\title{
The interaction of dyons in the mean field approximation
}

\author{
B.V.Martemyanov \\ Institute of Theoretical \& Experimental Physics \\ 117259, B.Cheremushkinskaya 25, Moscow, Russia
}

March 22, 2022

\begin{abstract}
The interaction of dyons in the mean field approximation is considered. The result of interaction is the mass term for dyonic field in the effective Lagrangian. Due to the mass term the profile function of dyon falls off exponentially at large distances.
\end{abstract}

\section{Introduction}

There is a hope that dyons, euclidean solutions of gauge theory, can be the fluctuations of vacuum fields that are responsible for confinement [1-4]. If we consider the gas of dyons (the simple superposition of dyonic solutions), neglect the interaction of dyons and omit their possible interference in the contribution to the Wilson loop we will get the phenomenon of "superconfinement" [2-4]. The "superconfinement" means that the Wilson loop average $W$ follows not the area low (confinement)

$$
W \sim \exp \left(-C r^{2}\right)
$$

where $C$ is some constant, $r$ is the radius of the loop (the circle, for example), but falls off like $\exp \left(-C^{\prime} r^{3}\right)\left(C^{\prime}\right.$ is the constant). It was put forward the idea [2-4] that due to the interaction of dyons something like "Debye screening" should appear in the dyonic gas transforming the "superconfinement" to confinement. In this paper we will consider the interaction of dyons in the mean field approximation and argue that effectively at large distances the dyonic field is exponentially damped. Such a damping is known for a long time in the case of instanton gas model of vacuum [5], where the damping was obtained from the Feynmann variational principle. We will consider the connection of the mean field approximation to the Feynmann variational principle. Now we are not in a position to say whether this damping is the desired "Debye screening" or not. In this sense the problem needs further investigation.

\section{Mean field approximation}

Let us consider the gas of dyons: $N$ dyons in the volume $V$. Dyons are described by their degrees of freedom: positions, color orientations (we consider $S U(2)$ gauge theory), 
"velocities". We consider one isolated dyon in the field of other dyons and average the total action over their degrees of freedom. In this way we can obtain the effective action for the dyon. Taking the minimum of the effective action we can find the modified dyonic solution. And at last we can use this modified solution to calculate some parameters of the effective action.

The described procedure is usually called the mean field approximation. We will show further the connection of this procedure with some form of the Feynmann variational principle. And now let us describe the details.

The total gauge field $A_{\mu}(x)=a_{\mu}(x)+B_{\mu}(x)$, where $a_{\mu}(x)$ is the gauge field of the isolated dyon and $B_{\mu}(x)$ is the field created by other dyons. The total action is equal to

$$
S=\int d^{4} x \frac{1}{4}\left(f_{\mu \nu}^{a}+F_{\mu \nu}^{a}+f_{\mu \nu}^{i n t, a}\right)^{2}
$$

where $f_{\mu \nu}^{i n t, a}=g \epsilon^{a b c}\left(a_{\mu}^{b} B_{\nu}^{c}+B_{\mu}^{b} a_{\nu}^{c}\right), f_{\mu \nu}$ and $F_{\mu \nu}$ are field tensors for potentials $a_{\mu}$ and $B_{\mu}$ respectively. The averaging of $S$ over the field $B_{\mu}$ is rather simple and as a result we get the effective action $\left(S_{\text {eff }}\right)$ for the isolated dyon

$$
S_{\text {eff }}=\int d^{4} x\left(\frac{1}{4} f_{\mu \nu}^{2}+\frac{m^{2}}{2} a_{\mu}^{2}\right)+S_{B}
$$

where $m^{2}=\frac{g^{2}}{2}<B_{\mu}^{2}>, S_{B}=\frac{1}{4}<F_{\mu \nu}^{2}>$. The constant $S_{B}$ is inessential for the calculation of the modified one-dyon solution.

The effective action is no more guage invariant and this is the reflection of the fact that the interaction of dyons depend in the model (simple superposition of individual dyons) on the gauge used for individual dyonic field [2]. The problem here is that the superposition of dyon's potentials is not only the solution of Yang-Mills equations but also gives the resulting field tensor depending on the gauge we are using for the dyon solution (the sum of potentials in one gauge is not connected to that in another gauge by any gauge transformation). The gauge should be taken in such a form as to get a minimal interaction of dyons (at large distances, at least). The interaction of dyons at large distances depends crucially on the asymptotic behaviour of dyonic potential and the latter depends on the class of the gauge, the different classes being connected by singular gauge transformations. In ref.[2] it was pointed out that the interaction of two dyons vanishing at infinity can be obtained in the so called 't Hooft gauge. We will use this gauge here also. We use the 't Hooft gauge $\left(\partial_{\mu} a_{\mu}=0\right)$ for the individual dyon solution in the superposition ansatz also for the following reasons. First, the topological charges of the dyons are summed in the considered (singular) gauge. Second, the interaction of dyons is minimal in this gauge (at least at infinitisimal gauge transformation). And third, because of the screened (due to the mass term) dyon solution satisfies the condition $\partial_{\mu} a_{\mu}=0$ automatically and transforms to the unscreened solution in the $m^{2} \rightarrow 0$ limit, the unscreened solution should satisfy the condition $\partial_{\mu} a_{\mu}=0$ also.

In the 't Hooft gauge the unscreened dyon solution is equal to

$$
\begin{gathered}
g a_{i}^{a}=-\epsilon_{i a b} n_{b} f(r, t)+\delta_{i}^{a} g(r, t) \\
g a_{0}^{a}=-n^{a} f(r, t) \\
f(r, t)=\frac{1}{r}+\gamma\left(\frac{\sinh \gamma r}{\cosh \gamma r-\cos \gamma t}-\frac{\cosh \gamma r}{\sinh \gamma r}\right)
\end{gathered}
$$




$$
g(r, t)=\gamma \frac{\sin \gamma t}{\cosh \gamma r-\cos \gamma t}
$$

Here $\gamma$ is the inverse size of the dyon. The mass term in the effective action (2) modifies the solution (3). If $m<<\gamma$ (the limit of pointlike dyons) qualitatively we can say that the internal part of the dyon is mainly unchanged (the function $g$ and the last two terms of function $f$ in (3) are unchanged) and the first term of function $f$ (Coulomb-like tail) $\frac{1}{r}$ transforms to $\frac{\exp (-m r)}{r}$ at $r>>\frac{1}{m}$. So, we have obtained the exponential damping of the dyonic solution at large distances. Obviously, the problem needs further numerical consideration at this point. The parameter $m$ of the effective action (2) can be now calculated using the modified one-dyon solution.

According to eqs.(2),(3)

$$
\begin{gathered}
m^{2}=(N-1) \frac{1}{2} g^{2}<a_{\mu}^{2}>= \\
=\frac{(N-1)}{V} 4 \pi \frac{3}{2} \int r^{2} d r\left(f^{2}+g^{2}\right) .
\end{gathered}
$$

In the limit of pointlike dyon we have approximately

$$
m^{2} \approx n 4 \pi \frac{3}{2} \frac{1}{2 m},(m<<\gamma)
$$

where $n=\frac{N}{V}$ is 3-d density of dyons.

For the unscreened solution the integral in formular (4) is divergent. Eq.(5) has the selfconsistent solution

$$
m={ }^{3} \sqrt{3 \pi n}
$$

\section{The connection to the Feynmann variational prin- ciple.}

The modification of the dyon solution can be also found by Feynmann variational principle. Let us assume that we are calculating the partition function $Z$ for $N$ dyons:

$$
Z=\int \exp (-S)
$$

where the integral goes over the dyons degrees of freedom. According to Feynmann variational principle

$$
Z \geq Z_{1} \exp \left(-<S-S_{1}>\right)
$$

where $S_{1}$ and $Z_{1}$ are the simplified action and the partition function. If we choose the action $S_{1}$ as a sum of $N$ independent (no interaction) terms: $S_{1}=N s_{1}$ then

$$
\begin{gathered}
Z_{1}=\int \exp \left(-N s_{1}\right)=A \exp \left(-N s_{1}\right) \\
<S_{1}>=N s_{1}
\end{gathered}
$$

where $A$ is the volume of the space of dyons degrees of freedom. So, we get

$$
Z \geq A \exp (-<S>)
$$


For $N$ dyons $\langle S>$ is equal to

$$
<S>=N \int d^{4} x \frac{1}{4} f_{\mu \nu}^{2}+\frac{1}{4} \frac{N(N-1)}{2} g^{2}<a_{\mu}^{2}>\int d^{4} x a_{\mu}^{2}
$$

The variation of $\left\langle S>\right.$ over $a_{\mu}$ (we are looking for the maximum of $Z$ and minimum of $<S>$ ) gives the same equation as the variation of $S_{\text {eff }}$ over $a_{\mu}$. The mass parameter $m^{2}$ is equal to

$$
m^{2}=(N-1) \frac{1}{2} g^{2}<a_{\mu}^{2}>
$$

in agreement with the result (4) obtained in the mean field approximation. We have applied the mean field approximation to the case of instantons (with fixed size $\rho$ for example). The mass term for the instanton field calculated in such a way coinsides with that of ref.[5] where the same problem was considered in the context of Feynmann variational principle.

\section{Conclusion}

We have considered the problem of the dyons interaction in the mean field approximation. In this approximation the interaction is effectively taken into account in the form of mass term for the dyonic field. The mass parameter $(m)$ is selfconsistently determined by the density of dyons (see eq.(5)). If other fluctuations (not of dyonic type) are present in the vacuum they also contribute to $m^{2}$. So, $m$ is larger than $\sqrt[3]{3 \pi n}$. The effect of the considered mass term is the exponential damping of the dyonic field at large distances.

This work is partly supported by the RFFI grants 96-02-00088G and 97-02-17491. The author would like to thank Yu.A.Simonov for stimulating discussions.

\section{References}

1. Yu.A.Simonov, Sov.J.Yad.Fiz. 43 (1985) 557.

2. Martemyanov B.V., Molodtsov S.V., Simonov Yu.A. and Veselov A.I., Pis'ma Zh.Eksp.Teor.Fiz. 62 (1995) 695

3. Martemyanov B.V., Molodtsov S.V.,Sov.J.Yad.Fiz. 59 (1996) 766.

4. Martemyanov B.V., Molodtsov S.V., Simonov Yu.A. and Veselov A.I.,Sov.J.Yad.Fiz. (1997) (to be published).

5. D.I.Diakonov and V.Yu.Petrov, Nucl.Phys. B245 (1984) 259. 